\title{
Thermal instability of implanted Mn ions in ZnO
}

\author{
J. A. Sans, ${ }^{1, a)}$ G. Martínez-Criado, ${ }^{1}$ J. Susini, ${ }^{1}$ R. Sanz,${ }^{2}$ J. Jensen, ${ }^{3}$ I. Minguez, ${ }^{2}$ \\ M. Hernandez-Velez, ${ }^{4}$ A. Labrador, ${ }^{5}$ and P. Carpentier ${ }^{6}$ \\ ${ }^{1}$ European Synchrotron Radiation Facility (ESRF), F-38043 Grenoble, France \\ ${ }^{2}$ Instituto de Ciencia de Materiales de Madrid (CSIC), Carretera de Colmenar Viejo, Cantoblanco, \\ 28049 Madrid, Spain \\ ${ }^{3}$ Thin Film Physics, Department of Physics, Chemistry and Biology, Linköping University, SE-581 83 \\ Linköping, Sweden \\ ${ }^{4}$ Departamento de Fisica Aplicada, Facultad de Ciencias, Campus Universitario de Cantoblanco, \\ 28049 Madrid, Spain \\ ${ }^{5}$ BM16-CRG, Consorci Laboratori de Llum de Sincrotró (LLS), clo ESRF, F-38043 Grenoble, France \\ ${ }^{6}$ Institut de Biologie Structurale, CNRS/CEA/UJF, F-38027 Grenoble, France
}

(Received 30 June 2009; accepted 24 November 2009; published online 25 January 2010)

\begin{abstract}
This letter reports on the site configuration of implanted Mn cations in $\mathrm{ZnO}$. The samples studied were obtained by means of ion implantation in m-plane $\mathrm{ZnO}$ single crystals. Synchrotron radiation based fluorescence shows no contamination during the implantation process. The results of micro-X-ray absorption spectroscopy indicate that $\mathrm{Mn}$ ions are located in substitutional sites without detectable traces of secondary phases. The postgrowth thermal annealing in $\mathrm{O}_{2}$ atmosphere induces a change in the coordination of a large amount of Mn cations, corresponding to $\alpha-\mathrm{Mn}_{2} \mathrm{O}_{3}$. (C) 2010 American Institute of Physics. [doi:10.1063/1.3275890]
\end{abstract}

\section{INTRODUCTION}

The study of alloys of $\mathrm{ZnO}$ with transition metals (TMs) have attracted a lot of interest in the past years due to its possible application in spintronic devices. The explanation of the origin of the magnetic properties, concretely ferromagnetism, in these wide band-gap materials has triggered the publication of a lot of papers with controversial results. These articles can be split into two categories depending on the local structure of TM in the wide band-gap material. On the one hand, considering the substitutional site of TM into the host lattice, ${ }^{1}$ several explanations had been proposed such as a hole mediated exchange Zener model published by Dietl et $a l^{2}$ or due to exchange mediated by carriers in a spin-split-orbit impurity band derived from extended orbitals in donor centers. ${ }^{3}$ On the other hand, the possible ferromagnetism is considered to come from clusters ${ }^{4}$ or segregation of different TM oxides. ${ }^{5-7}$

One of the most important factors in order to obtain reliable results is the growth method. In this framework, the ion implantation has shown several advantages ${ }^{8}$ with respect to other growth techniques, such as the control of the ion fluency and the use of an isotopically pure ionic beam, but also some disadvantages ${ }^{9}$ such as the damage produced on surface. Fortunately, the application of thermal annealing in oxidizing conditions reduces the implantation damage ${ }^{9,10}$ but in the case of alloys of $\mathrm{ZnO}$ with TM has shown a tendency to form TM oxides precipitates. ${ }^{8,11}$ Such new phases are very difficult to detect by means of x-ray diffraction, Raman, or high resolution electron microscopy due to the low amount of TM in the host sample. However, micro-X-ray absorption spectroscopy ( $\mu$ XAS) technique is an useful tool to elucidate the local coordination of these elements.

${ }^{\text {a)} E l e c t r o n i c ~ m a i l: ~ s a n s t r e s @ e s r f . f r . ~}$

\section{EXPERIMENT}

Small pieces of $\mathrm{ZnO}$ (wurtzite) single crystal (MTI Crystal Corp.) with polished m-plane surface orientation and a nominal density of $5.2 \mathrm{~g} \mathrm{~cm}^{-3}$ were used as substrates. The implantation process was performed at normal incidence with $35 \mathrm{keV} \mathrm{Mn}^{+}$ions, with a total fluency of 4.2 $\times 10^{16}$ ions $/ \mathrm{cm}^{2}$ and an average current density of $0.4 \mu \mathrm{A} \mathrm{cm}^{-2}$. Simulations of implantation profiles were carried out by the SRIM2006 code, ${ }^{12}$ suggesting a Gaussian distribution with a maximum concentration peak at $20 \mathrm{~nm}$ from the surface and a straggling of $10 \mathrm{~nm}$. After ion implantation these so-called continuum implanted samples were treated applying a rapid thermal annealing process in $\mathrm{O}_{2}$ atmosphere at $800{ }^{\circ} \mathrm{C}$ for $3 \mathrm{~min}$. $\mu \mathrm{XAS}$ and scanning $\mathrm{x}$-ray fluorescence (XRF) measurements were carried out at the microprobe station ID22 $2^{3,13}$ of the European Synchrotron Radiation Facility. The beamline is equipped with two insertion devices, a flat $\mathrm{Si}$ mirror at $0.14^{\circ}$ incident angle for harmonic rejection and a fixed-exit double $\mathrm{Si}(111)$ crystal monochromator $\left(\Delta \mathrm{E} / \mathrm{E} \sim 10^{-4}\right)$. The monochromatic beam is focused on the sample down to a spot of $1.5 \times 3.5 \mu \mathrm{m}^{2}$ size with a focus depth of $300 \mu \mathrm{m}$ by means of a pair of mirrors in Kirkpatrick-Baez geometry. The intensity of the incident $\mathrm{x}$-ray beam $\left(\sim 10^{11}\right.$ photon/s in the focused beam at $\mathrm{Mn}$ K-edge) was monitored with an ionization chamber, while a Si drift detector was used to collect the characteristic x-ray emission lines. In fluorescence detection mode, $\mu$ XAS measurement conditions at the $\mathrm{Mn}-\mathrm{K}$ edge included a photon energy range of $6500-6650 \mathrm{eV}$ and an energy step of $0.5 \mathrm{eV}$, and integration times determined by the counting statistics. XRF measurements were carried out with an incident angle of $\theta=45 \pm 5^{\circ}$ with respect to the sample surface to minimize the contribution of elastic scattering to the signal background. Micro-Raman spectra were recorded in Cry- 


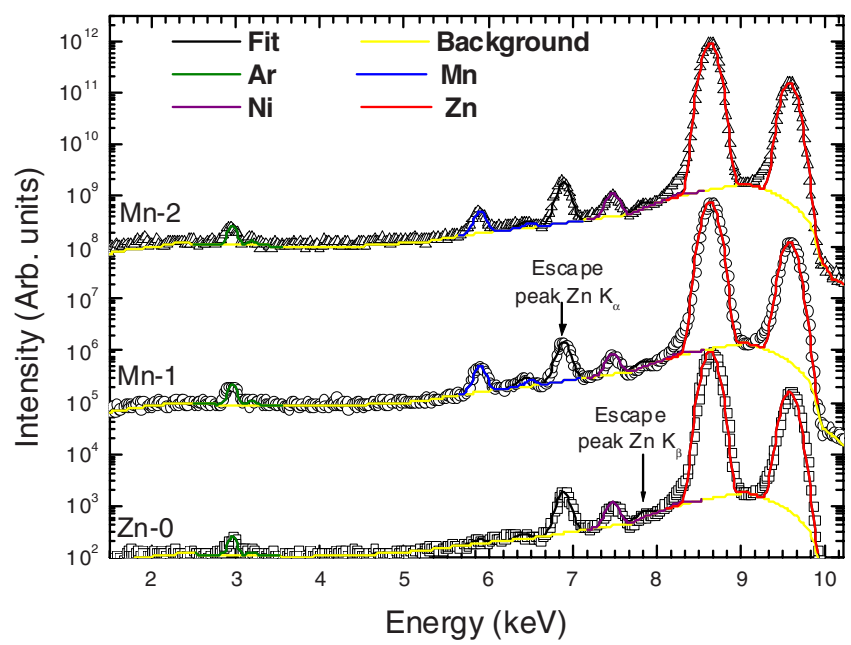

FIG. 1. (Color online) XRF spectra for $\mathrm{ZnO}$ single monocrystal $(\mathrm{Zn}-0)$, as-implanted $\mathrm{ZnMnO}(\mathrm{Mn}-1)$, and thermal annealed implanted $\mathrm{ZnMnO}$ (Mn-2), shifted for clarity.

obench Facility of ESRF with a $514 \mathrm{~nm}$ excitation line of an $\mathrm{Ar}+$ laser using a Renishaw Invia system ${ }^{14}$ equipped with a compact probe compatible with an x-ray goniometer. The Raman scattering experiments were performed in backscattering configuration with a spectral shift resolution better than $1 \mathrm{~cm}^{-1}$.

\section{RESULTS}

In this work $\mathrm{Zn}-0, \mathrm{Mn}-1$, and $\mathrm{Mn}-2$ labels correspond to $\mathrm{ZnO}$ single crystal, $\mathrm{Mn}$ implanted $\mathrm{ZnO}$, and thermal annealed $\mathrm{Mn}$ implanted $\mathrm{ZnO}$, respectively. The homogeneity of the Mn implantation was validated by measuring its fluorescence line intensity over $50 \times 50 \mu \mathrm{m}^{2}$ sample area (not shown), taking advantage of the piezoelectric ${ }^{1}$ scanning at the microprobe ID22. XRF measurements for $\mathrm{Zn}-0, \mathrm{Mn}-1$, and $\mathrm{Mn}-2$ are shown in Fig. 1 on logarithmic scale (vertically shifted for clarity). "Escape peaks" have been observed in these spectra, generated from the Si drift detector as the combination of a Si K shell transition with the most intense peaks (in this case the $\mathrm{Zn} \mathrm{K}$ energy peaks). The $12 \mathrm{keV}$ excitation energy allows to measure the $\mathrm{K}$ shell lines of $\mathrm{Zn}, \mathrm{Mn}, \mathrm{Ni}$, and Ar. The Ni traces are associated with the Zn-0 template and used as substrate for the implantation, and Ar line is originated from air. The implanted Mn concentration was calculated from the $\mathrm{Mn}$ and $\mathrm{Zn}$ shell line intensity ratio using PYMCA code,${ }^{15}$ giving around $0.2 \%$ of $\mathrm{Mn}$ in $\mathrm{Mn}-1$ and $\mathrm{Mn}-2$ samples. Within the detection limits of the technique, no traces of other elements were observed after implantation in the considered energy range. Thermal instability in oxidizing conditions is a crucial issue in future potential applications. It has already been reported in alloys or doped $\mathrm{ZnO}$, such as ZnO:Ga (Ref. 16) or $\mathrm{ZnFeO}{ }^{11}$

Raman spectroscopy is a powerful tool to measure the characteristic vibration modes of the lattice and symmetry dependent phonon modes. Since $\mathrm{ZnO}$ crystallizes in the wurtzite structure belonging to the $C 46 v(P 63 m c)$ space group, the zone center phonons are $2 \mathrm{~A} 1+2 \mathrm{~B} 1+2 \mathrm{E} 1+2 \mathrm{E} 2$, from which one $\mathrm{A} 1$, one $\mathrm{E} 1$, and two $\mathrm{E} 2$ are Raman active, whereas the B1 modes are silent. In addition, one set of A1
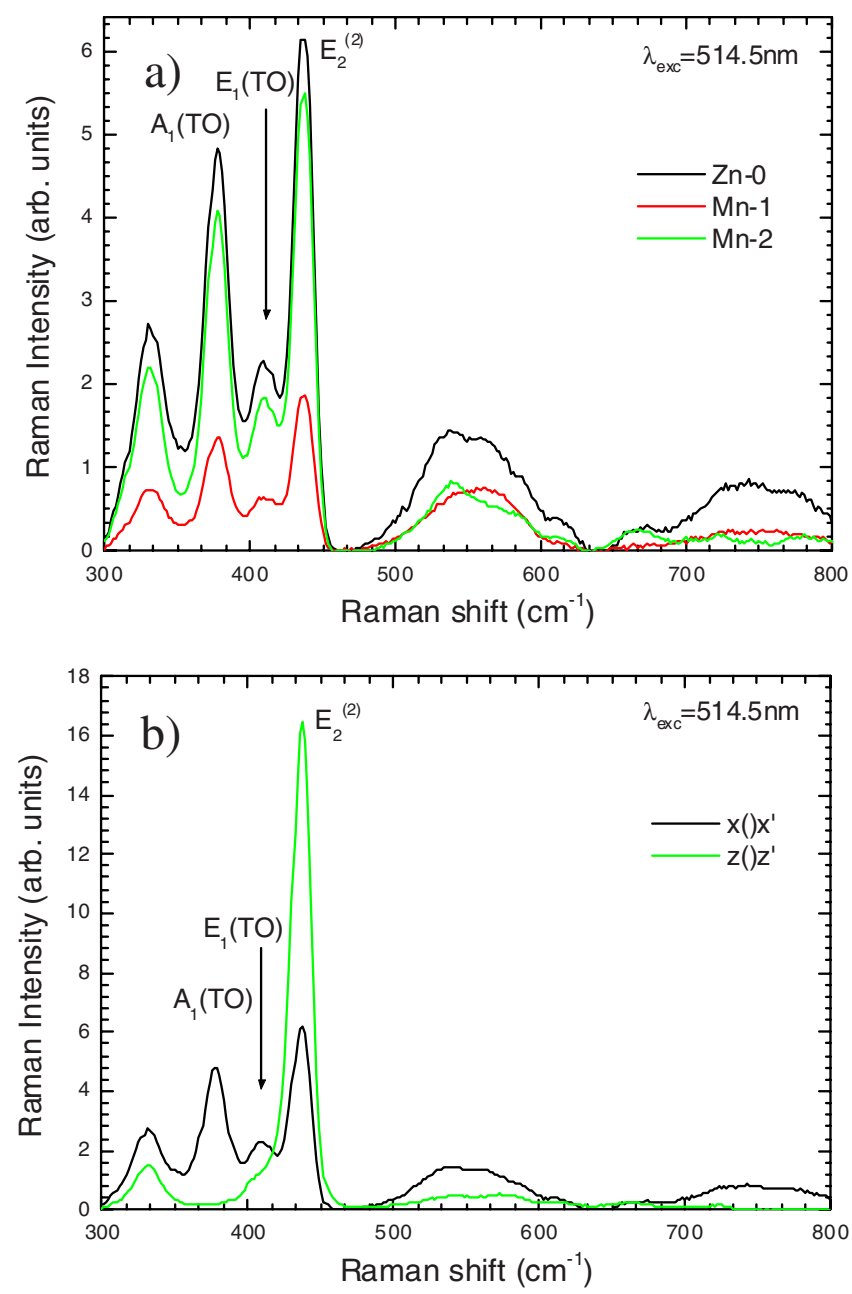

FIG. 2. (Color online) (a) Raman scattering spectra for samples Zn-0, Mn-1, and Mn-2. (b) Spectra of sample of Mn-1 for two configurations indicated in the figure.

and E1 corresponds to acoustic phonons. The anisotropic short-range order of the hexagonal structure is responsible for the A1-E1 splitting, whereas the long-range Coulomb field causes the polar modes split into longitudinal-transverse optical modes at the zone center. Figure 2 shows the Raman spectra for different configurations and samples. The findings indicate that the implanted elements are in substitutional site due to the absence of peaks related to other phases. ${ }^{9}$ No differences between $\mathrm{Zn}-0, \mathrm{Mn}-1$, and $\mathrm{Mn}-2$ spectra have been observed. Peaks located at 377,410 , and $437 \mathrm{~cm}^{-1}$ are assigned to $\mathrm{ZnO} \mathrm{A} 1(\mathrm{TO}), \mathrm{E} 1(\mathrm{TO})$, and E2(2) Raman peaks respectively ${ }^{17}$ and its appearance in spectra is determined by the selection rules of the wurtzite structure. ${ }^{18}$ The second order Raman peak measured at $332 \mathrm{~cm}^{-1}$ has been associated in the literature ${ }^{19}$ to the sum of two phonons coming from $\mathrm{K}-\mathrm{M}-\Sigma$ transition at $160 \mathrm{~cm}^{-1}$. Two major bands centered at 550 and $750 \mathrm{~cm}^{-1}$ with large bandwidths also correspond to overtones due to Fröhlich electron-phonon scattering. The effect of thermal annealing does not show any additional Raman peaks from secondary phases or disorder activated phonon modes. The presences of the peaks related to the Fröhlich electron-phonon scattering would hide the possible appearance of the characteristic Raman peak ${ }^{20}$ of $\mathrm{Mn}_{2} \mathrm{O}_{3}$, which is located at $680 \mathrm{~cm}^{-1}$. 


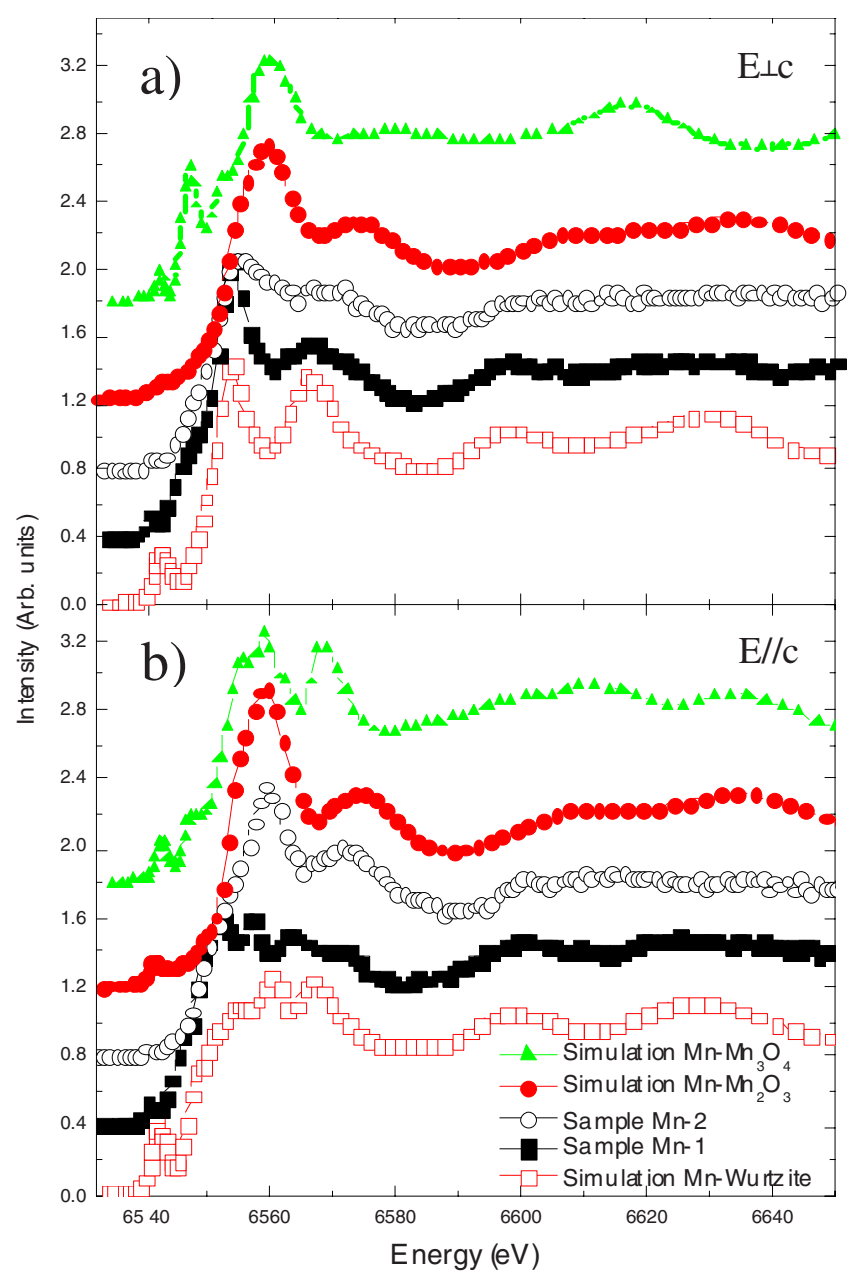

FIG. 3. (Color online) XANES data for samples Mn-1 and Mn-2. The figure also includes the theoretical simulations for $\mathrm{Mn}$ in substitutional site in $\mathrm{ZnO}$ and $\mathrm{Mn}$ in $\alpha-\mathrm{Mn}_{2} \mathrm{O}_{3}$ structure: (a) with electric field perpendicular to the c-axis and (b) with electric field parallel to c-axis.

Linear polarization dependence of the x-ray absorption near edge structure (XANES) spectra gives a measurement of preferential directions and anisotropies in the local coordination of an element into a lattice. Figure 3 shows the study of the variation in XANES spectra around Mn K-edge with the electrical field vector parallel and perpendicular to the c-axis of the $\mathrm{ZnO}$. The simulations performed at the $\mathrm{Mn}$ $\mathrm{K}$ edge using the $a b$ initio self-consistent FEFF8 $\operatorname{code}^{21}$ are also included in the figure for comparison. In our case, the anisotropy $^{22,23}$ of the wurtzite structure was also used to identify the influence of impurities into the local environment. Figure 3 displays the results obtained for sample Mn-1. The good agreement between the experimental Mn K-edge absorption spectrum and the simulation of $\mathrm{Mn}$ in substitutional $\mathrm{Zn}$ sites of $\mathrm{ZnO}$ shows that most of Mn cations are tetrahedrally coordinated by oxygen in sample Mn-1. Another indication of this fact is the strong resemblance between the $\mathrm{Mn}$ and $\mathrm{Zn} \mathrm{K}$-edges (not shown here). Small amounts of other phases cannot be completely neglected according to the sensitivity of this technique. For the parallel geometry $(\mathrm{E} \| \mathrm{c})$, for instance, apart from the most intense peaks located above the edge, the XANES spectrum of the as-implanted sample could look like to the one of $\mathrm{Mn}_{3} \mathrm{O}_{4}$ in the pre-edge and edge regions. However, the relative intensity of the different resonances, energy positions, as well as their polarization dependencies do not follow the same trend. The implication of $\mathrm{Mn}_{3} \mathrm{O}_{4}$ for such configuration (E\|c) was excluded based on linear combination analysis. Using the theoretical simulations of $\mathrm{Mn}$ with wurtzite and $\mathrm{Mn}_{3} \mathrm{O}_{4}$ coordinations, the resulting spectral shapes exhibit predominantly the dominant features at 6600 and $6626 \mathrm{eV}$ coming from the characteristic Mn cations in tetrahedral sites (wurtzite), with an unclear and unconvincing role played by the $\mathrm{Mn}_{3} \mathrm{O}_{4}$. As a result, the observations around the white line could be due to the damage produced in the implantation, which would produce slight changes in the lineshape of the spectrum, namely, structural disorder around the Mn ion and/or a modification of the p-electronic density caused by defects $^{24}$ (vacancies, interstitial ions, etc.). The slight difference observed of the lineshape around the white line could be explained taking into account the damage produced in the implantation. This effect has already been studied for other implanted samples like cubic-BN, ${ }^{25}$ where a change around the white line was observed with the variation in the kinetic energy of the implanted ions.

Figure 3 also displays the XANES spectra for sample Mn-2. The net difference between the experimental XANES spectra observed for the as-implanted sample and the annealed one could be considered as a fingerprint of a phase transition of a large amount of the $\mathrm{Mn}$ atoms. This change in the local coordination of Mn cations is related to the application of a thermal annealing in $\mathrm{O}_{2}$ atmosphere. An oxygenrich atmosphere in the thermal annealing would produce the formation of oxygen-richer structures such as spinels or certain Mn oxide phases. A comparison between the experimental data and several theoretical simulations was carried out in order to determine the crystalline structure formed $\left(\mathrm{Mn}_{3} \mathrm{O}_{4}\right.$, $\mathrm{Mn}_{2} \mathrm{O}_{3}$, etc.). The results show that the change observed in the absorption spectrum of Mn K-edge in sample Mn-2 corresponds to the formation of $\alpha-\mathrm{Mn}_{2} \mathrm{O}_{3}$ phase. Accordingly, the oxidation number of $\mathrm{Mn}$ shifts from +2 to +3 in a cubic bixbyite crystalline structure, where Mn would be octohedrally coordinated by oxygen. The diffusion of $\mathrm{Zn}$ into this phase was proposed ${ }^{26}$ as a possible explanation for the origin of the ferromagnetism at room temperature observed in the literature. Nevertheless, the broad peaks located at 6600 and $6626 \mathrm{eV}$ are characteristic features of wurtzite structure, which would indicate that a certain proportion of Mn atoms remain in substitutional position after the thermal annealing. Our finding is an interesting result, taking into account that the coexistence of ions with different oxidation number $\mathrm{Mn}^{2+}$ and $\mathrm{Mn}^{3+}$ has been proposed to play an important role in the ferromagnetism observed in GaMnN. ${ }^{27}$ Some studies have extended this model to other wide band-gap TM alloys, ${ }^{27}$ such as $\mathrm{ZnMnO}$.

Figure 4(a) displays the X-ray Linear dichroism (XLD) signal for as-implanted sample (Mn-1), where the experimental spectrum fits to the theoretical simulation for $\mathrm{Mn}$ in wurtzite structure. As a matter of fact, this result supports the substitutional character of $\mathrm{Mn}$ into the $\mathrm{ZnO}$ host lattice. The XLD signal for the thermal annealed sample in oxidizing conditions (Mn-2) is shown in Fig. 4(b). The experimental 

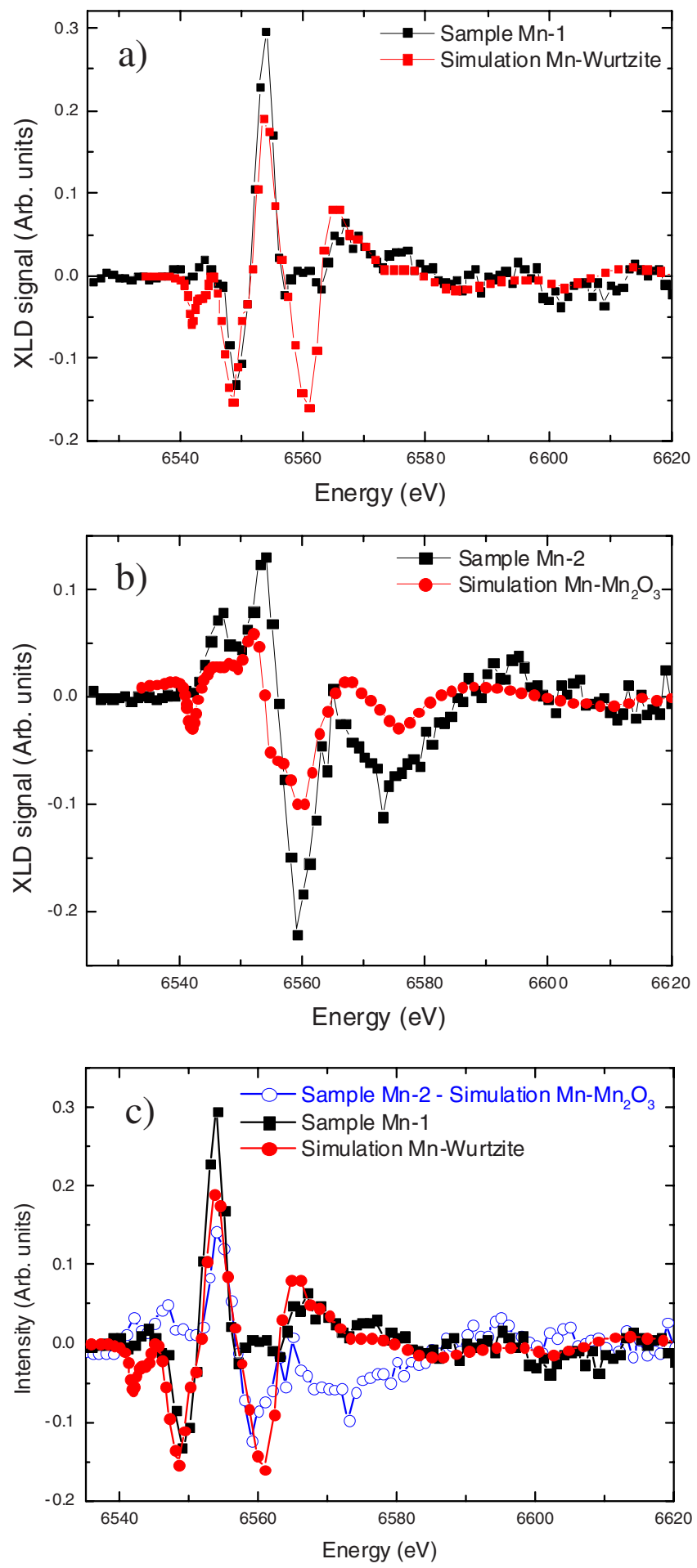

FIG. 4. (Color online) XLD signal obtained from (a) sample Mn-1 and theoretical simulation of $\mathrm{Mn}$ in wurtzite structure, (b) sample Mn-2 and theoretical simulation of $\mathrm{Mn}$ in $\alpha-\mathrm{Mn}_{2} \mathrm{O}_{3}$ phase, and (c) subtraction between the theoretical simulation of $\mathrm{Mn}_{2} \mathrm{O}_{3}$ and the signal corresponding to the sample Mn-2 compared with XLD signal from Mn-1 sample. The simulation signal of $\mathrm{Mn}$ in wurtzite structure has been also included for comparison.

data present a similar spectral shape compared with the theoretical simulation for $\mathrm{Mn}$ in $\alpha-\mathrm{Mn}_{2} \mathrm{O}_{3}$ phase. Nevertheless, a shoulder appears in the main peak, which would reveal the presence of Mn cations in a different crystalline structure. To explain this phenomenon, the theoretical XLD signal for Mn forming $\mathrm{Mn}_{2} \mathrm{O}_{3}$ was subtracted to the experimental one of the sample $\mathrm{Mn}-2$ in order to distinguish the contribution of each different phase. A similar analysis was not doable for the unannealed sample because of the poor signal-to-noise ratio of the corresponding difference spectrum. In the annealed case, the resulting curve was compared to the experimental XLD signal obtained for sample Mn-1 [Fig. 4(c)]. The energy position of the dominant peak is equivalent for both signals $(6554 \mathrm{eV})$. Regardless the signal amplitude, the correspondence could indicate that a certain amount of $\mathrm{Mn}$ cations remains in wurtzite structure even after the thermal annealing in oxidizing conditions, coherently with the assumptions done in the analysis of the polarization dependent XANES spectra. The ratio between the intensities of the main peak would allow to determine roughly the relative proportion of Mn cations remaining in substitutional site into $\mathrm{ZnO}$ host lattice respect to the implanted sample. Our calculation works out that $\sim 50 \%$ of Mn remains in wurtzite structure after the thermal annealing.

\section{CONCLUSIONS}

In summary, the structural properties of Mn implanted $\mathrm{ZnO}$ samples and its thermal stability was investigated by a multimodal analysis using $\mathrm{x}$-ray microspectroscopic techniques. XRF measurements showed no traces of contamination during the growth process. Raman spectra exhibited the unambiguous signature of the $\mathrm{ZnO}$ wurtzite structure without secondary phases even after the thermal annealing. XANES and XLD measurements revealed $\mathrm{Mn}$ ions in substitutional sites in the $\mathrm{ZnO}$ host lattice for as-implanted sample. A phase transition is produced in a large amount of $\mathrm{Mn}$ atoms after the thermal annealing in oxidizing conditions. Moreover, the coexistence of $\mathrm{Mn}$ in wurtzite structure and $\mathrm{Mn}$ in a new crystalline structure was presented and tentatively attributed to the segregation of $\mathrm{Mn}$ in $\alpha-\mathrm{Mn}_{2} \mathrm{O}_{3}$ phase.

\section{ACKNOWLEDGMENTS}

The authors thank to Dr. G. Gonzalez for providing the implantation system and Professor M. Vazquez for financial support.

${ }^{1}$ T. Dietl, H. Ohono, F. Matsukura, J. Cibert, and D. Ferrand, Science 287, 1019 (2000).

${ }^{2}$ G. Martinez-Criado, A. Segura, J. A. Sans, A. Homs, J. Pellicer-Porres, and J. Susini, Appl. Phys. Lett. 89, 061906 (2006)

${ }^{3}$ J. M. D. Coey, M. Venkatesan, and C. B. Fitzgerald, Nature Mater. 4, 173 (2005).

${ }^{4}$ J. H. Park, M. G. Kim, H. M. Jang, S. Ryu, and Y. M. Kim, Appl. Phys. Lett. 84, 1338 (2004).

${ }^{5}$ S. Kolesnik, B. Dabrowski, and J. Mais, J. Appl. Phys. 95, 2582 (2004).

${ }^{6}$ D. P. Norton, M. E. Overberg, S. J. Pearton, K. Pressner, J. D. Budai, L. A. Boatner, M. F. Chisholm, J. S. Lee, Z. G. Khim, Y. D. Park, and R. G. Wilson, Appl. Phys. Lett. 83, 5488 (2003).

${ }^{7}$ T. Dietl, T. Andrearczyk, A. Lipinska, M. Kiecana, M. Tay, and Y. Wu, Phys. Rev. B 76, 155312 (2007)

${ }^{8}$ S. Zhou, K. Potzger, G. Talut, H. Reuther, J. von Borany, R. Grotzschel, W. Skorupa, M. Helm, and J. Fassbender, J. Appl. Phys. 103, 023902 (2008).

${ }^{9}$ M. Schumm, M. Koerdel, S. Muller, H. Zutz, C. Ronning, J. Stehr, D. M. Hofmann, and J. Geurts, New J. Phys. 10, 043004 (2008).

${ }^{10}$ S. Ramachandran, J. Narayan, and J. T. Prater, Appl. Phys. Lett. 88, 242503 (2006)

${ }^{11}$ S. Zhou, K. Potzger, J. von Borany, R. Grotzschel, W. Skorupa, M. Helm, and J. Fassbender, Phys. Rev. B 77, 035209 (2008).

${ }^{12}$ www.srim.org. 
${ }^{13}$ G. Martinez-Criado, A. Somogyi, S. Ramos, J. Campo, R. Tucoulou, M. Salome, J. Susini, M. Hermann, M. Eickhoff, and M. Stutzmann, Appl. Phys. Lett. 86, 131927 (2005)

${ }^{14} \mathrm{P}$. Carpentier, A. Royant, J. Ohana, and D. Bourgeois, J. Appl. Crystallogr. 40, 1113 (2007).

${ }^{15}$ V. A. Solé, E. Papillon, M. Cotte, Ph. Walter, and J. Susini, Spectrochim. Acta, Part B 62, 63 (2007)

${ }^{16}$ J. A. Sans, G. Martinez-Criado, J. Pellicer-Porres, J. F. Sanchez-Royo, and A. Segura, Appl. Phys. Lett. 91, 221904 (2007)

${ }^{17}$ F. Decremps, J. Pellicer-Porres, A. Marco Saitta, J.-C. Chervin, and A. Polian, Phys. Rev. B 65, 092101 (2002).

${ }^{18}$ P. Dahan, V. Fleurov, and K. Kikoin, J. Phys.: Condens. Matter 9, 5355 (1997).

${ }^{19}$ J. M. Calleja and M. Cardona, Phys. Rev. B 16, 3753 (1977).

${ }^{20}$ F. Buciuman, F. Patcas, R. Craciun, and D. R. T. Zahn, Phys. Chem. Chem. Phys. 1, 185 (1999).
${ }^{21}$ A. L. Ankudinov, B. Ravel, J. J. Rehr, and S. D. Conradson, Phys. Rev. B 58, 7565 (1998).

${ }^{22}$ G. Martínez-Criado, O. Sancho-Juan, N. Garro, J. A. Sans, A. Cantarero, J. Susini, M. Roever, D.-D. Mai, A. Bedoya-Pinto, J. Malindretos, and A. Rizzi, Appl. Phys. Lett. 93, 021916 (2008).

${ }^{23}$ F. J. Manjon, D. Errandonea, A. H. Romero, N. Garro, J. Serrano, and M. Kuball, Phys. Rev. B 77, 205204 (2008).

${ }^{24}$ C. Mignote, Nucl. Instrum. Methods Phys. Res. B 187, 95 (2002).

${ }^{25}$ R. Gago, B. Abendroth, J. I. Cerda, I. Jimenez, and W. Moller, Phys. Rev. B 76, 174111 (2007).

${ }^{26}$ D. C. Kundaliya, S. B. Ogale, S. E. Lofland, S. Dhar, C. J. Metting, S. R. Shinde, Z. Ma, B. Varughese, K. V. Ramanujachary, L. Salamanca-Riba, and T. Venkatesan, Nature Mater. 3, 709 (2004).

${ }^{27}$ S. Sonoda, I. Tanaka, H. Ikeno, T. Yamamoto, F. Oba, T. Araki, Y. Yamamoto, K. Suga, Y. Nanishi, Y. Akasaka, K. Kindo, and H. Hori, J. Phys.: Condens. Matter 18, 4615 (2006) 